\title{
Comparison of plasma to tissue DNA mutations in surgical patients with non-small cell lung cancer
}

\author{
Kezhong Chen, MD, ${ }^{\mathrm{a}}$ Jingbo Zhang, MS, ${ }^{\mathrm{b}}$ Tian Guan, MD, ${ }^{\mathrm{a}}$ Fan Yang, MD, ${ }^{\mathrm{a}}$ Feng Lou, PhD, \\ Wei Chen, MS, ${ }^{b}$ Mingyu Zhao, MS, ${ }^{b}$ Jay Zhang, PhD, ${ }^{b}$ Siyi Chen, $\mathrm{PhD},{ }^{\mathrm{c}}$ and Jun Wang, $\mathrm{MD}^{\mathrm{a}}$
}

\section{ABSTRACT}

Objective: Noninvasive liquid biopsies of circulating tumor DNA (ctDNA) can be used to assess non-small cell lung cancer (NSCLC), but previous work focused on patients with advanced-stage cancer. Thus, we evaluated the feasibility and their potential clinical application of circulating tumor DNA approached for surgical patients with NSCLC.

Method: Consecutive patients with suspected lung cancer who underwent curative-intent lung resection were enrolled prospectively in this study. Targeted DNA sequencing with a next-generation sequencing platform was used to identify a series of somatic mutations in matched tumor tissue DNA (tDNA) and plasma ctDNA samples. Plasma was collected before, during, and after surgery. Concordance was defined as matched tDNA and ctDNA with the same identified mutations or with no mutations.

Results: In the enrolled 76 patients with lung cancer who were included, 31 had concordant mutations and 21 had no mutation in both ctDNA and tDNA, yielding an overall concordance of $68.4 \%$. ctDNA samples obtained before and during surgery had the same mutations with a low variance in mutation frequency $(1.2 \%)$ that was reduced to an average of $0.28 \%$ after surgery $(P<.001)$. More patients were positive as assayed by ctDNA $(48 ; 63.2 \%)$ than with serum tumor protein markers $(36 ; 49.3 \%)$. The area under the curve was greater in ctDNA $(0.887$, $95 \%$ confidence interval [CI], 0.788-0.986) than for the 2 prediction models $(0.803,95 \%$ CI, 0.647-0.959; 0.69, 95\% CI, 0.512-0.869) for estimating malignancy of solitary pulmonary nodules.

Conclusion: ctDNA mutation analysis for stage I-III surgical patients with NSCLC is feasible. More studies are needed to investigate its clinical application. (J Thorac Cardiovasc Surg 2017;154:1123-31)

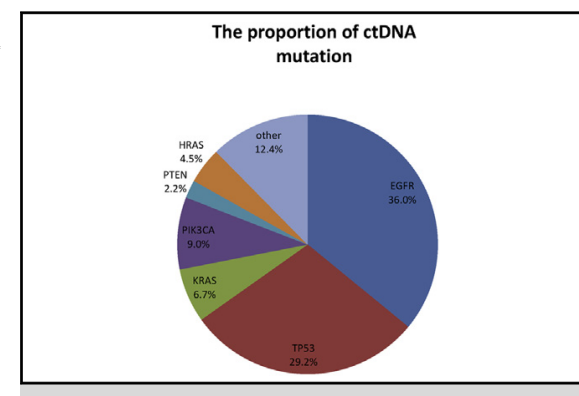

Gene mutations identified in plasma circulating tumor DNA.

\section{Central Message}

Plasma circulating tumor DNA multiple mutation analysis in stage I-III, surgical patients is feasible and has potential clinical value.

\section{Perspective}

Most previous studies of circulating tumor DNA focused on advanced stage non-small cell lung cancer. This prospective study shows multiple gene detection using plasma circulating tumor DNA in surgical non-small cell lung cancer patients is feasible and has potential clinical application. Further studies are needed before it is brought into mainstream clinical practice.

See Editorial Commentary page 1132.
Circulating tumor DNA (ctDNA) presents a noninvasive liquid biopsy and is theoretically reflective of the total underlying tumor burden. ${ }^{1}$ Thus, identifying ctDNA offers

\footnotetext{
From the a Department of Thoracic Surgery, Peking University People's Hospital; ${ }^{\mathrm{b}}$ San Valley Biotechnology Inc, Beijing, China; and ${ }^{\mathrm{c}}$ Norris Comprehensive Cancer Center, Department of Molecular Microbiology and Immunology, Keck School of Medicine, University of Southern California, Los Angeles, Calif. This study was supported by the National Natural Science Funds (No.81602001) and Peking University People's Hospital Research and Development Funds (RDY2016-03)

Read at the 96th annual meeting of The American Association for Thoracic Surgery, Baltimore, Maryland, May 14-18, 2016.

Received for publication May 26, 2016; revisions received April 7, 2017; accepted for publication April 18, 2017; available ahead of print June 16, 2017.

Address for reprints: Jun Wang, MD, Department of Thoracic Surgery, Peking University People's Hospital, Beijing 100044, China (E-mail: wangjun@pkuph.edu.cn). $0022-5223 / \$ 36.00$

Copyright (c) 2017 by The American Association for Thoracic Surgery http://dx.doi.org/10.1016/j.jtcvs.2017.04.073
}

the opportunity to assist diagnosis, detect molecular changes, monitor disease progress, and predict treatment response. $^{2,3}$ However, most previous studies of ctDNA have focused on advanced stage and metastatic cancers (stage IIIB-IV) with a limited number of driver mutations. ${ }^{4,5}$ Few data are available to indicate whether ctDNA analyses is applicable for patients with stage I-IIIA nonsmall cell lung cancer (NSCLC), largely because the low

Scanning this QR code will take you to a supplemental video, tables, and figures. To view the AATS 2016 Webcast, see the URL next to the webcast thumbnail. 


\section{Abbreviations and Acronyms \\ $\mathrm{CI}=$ confidence interval \\ $\mathrm{CT}=$ computed tomography \\ ctDNA = circulating tumor DNA \\ EGFR = epidermal growth factor receptor \\ NSCLC $=$ non-small cell lung cancer \\ SPN $=$ solitary pulmonary nodule \\ tDNA $=$ tumor DNA}

level of micrometastatic disease makes detection of ctDNA challenging. ${ }^{6}$

Next-generation sequencing using the Ion Personal Genome Machine has been shown previously to have high sensitivity and can screen for mutations in multiple genes simultaneously, ${ }^{7}$ which may be useful for identifying plasma ctDNA from patients with lung cancer. Thus, our prospective study evaluated the feasibility of identifying ctDNA in plasma of surgical patients with NSCLC with matched tumor tissue using targeted sequencing and investigated potential clinical applications.

\section{METHODS}

We prospectively enrolled consecutive patients who underwent curativeintent lung resection between May and July 2015. Patients with malignant tumor histories or who received treatment before resection were excluded. One hundred ten patients met the inclusion criteria and were collected blood samples during surgery, before the tumor was resected (Video 1). ctDNA was extracted immediately to avoid DNA degradation. In an initial 24 patients, plasma 1 to 3 days before surgery and 1 day after surgery was obtained to confirm method reproducibility and to investigate dynamic changes in ctDNA.

Tumor samples were collected during surgery when a frozen section was confirmed as malignant. Fifteen patients had benign lesions as confirmed with frozen sections, and 95 patients were enrolled and were processed for tumor DNA extraction before pathology and staging were known. Peripheral blood cells collection was not achieved for 2 samples, and ctDNA extraction failed for 3 samples ( 2 serum; 1 tissue sample). Later, formalin-fixed, paraffin-embedded sections indicated 3 small cell carcinomas, 1 carcinoid, and 2 unclassified carcinomas, which were excluded from the analysis. For the 84 patients who had paired samples, 8 patients with ground-glass opacity (GGO) with adenocarcinoma in situ or minimally invasive adenocarcinoma were excluded from this study because of concerns about tumor cell proportion. In total, 76 patients diagnosed with stage IA-IIIA adenocarcinoma or squamous cell carcinoma were included to compare tumor DNA and plasma ctDNA (Figure 1). A study protocol (ClinicalTrials.gov identifier NCT02645318) was approved by the Institutional Review Board of Peking University People's Hospital (2015PHB055-01). Written informed consent was obtained from all donors.

\section{Sample Preparation and Sequencing}

Fresh tumor tissue, white blood cells, and plasma samples were collected from each patient. Tumor tissue was resected from the tumor core area, half of which was sent for frozen pathologic examination to confirm lung cancers; the other portion was used for DNA extraction. Peripheral blood samples $(10 \mathrm{~mL})$ were obtained at each time point and processed immediately to isolate plasma from ethylenediaminetetraacetic acid tubes. Samples were centrifuged for 10 minutes at $1600 g$, and peripheral blood lymphocyte debris was stored at $-20^{\circ} \mathrm{C}$ until use. Supernatants from these samples were again centrifuged at
$16,000 \mathrm{~g}$ for 10 minutes, and plasma was collected and stored at $-80^{\circ} \mathrm{C}$ until needed. DNA from fresh tissue, peripheral blood lymphocytes, and ctDNA from plasma were extracted separately. A Qubit 2.0 Fluorometer and Qubit dsDNA HS Assay kit (Life Technologies, Carlsbad, Calif) was used according the manufacturer's instructions. Ion Personal Genome Machine library preparation, sequencing, and variant calling were performed as previously described. $^{7,8}$ A panel of 50 cancer-related genes was assayed with targeted sequencing. White blood cells from each patient were analyzed as controls. These methods are depicted in Figure E1.

\section{Clinical Prediction Model}

Preoperative precise diagnosis of solitary pulmonary nodule (SPN) is important for clinical decision-making, but SPN often yields atypical imaging findings. Traditionally, preoperative assessment of SPN is based on clinician and radiologist expertise or with a mathematical prediction model. ${ }^{9}$ The most widely used model (the Mayo model) reported by Swensen and colleagues ${ }^{10}$ suggests that the capacity for judging SPN characteristics is similar to clinical judgment of experienced physicians. We established the first Chinese SPN prediction model (PKU model) in 2011, which may be more applicable for those of Asian heredity. ${ }^{11}$ These 2 models are as follows:

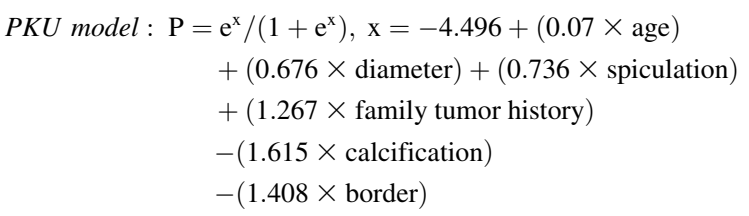

Mayo model: $P=\mathrm{e}^{\mathrm{x}} /\left(1+\mathrm{e}^{\mathrm{x}}\right), \mathrm{x}=-6.8272+(0.0391 \times$ age $)$

$$
\begin{aligned}
& +(0.7917 \times \text { smoking history }) \\
& +(1.3388 \times \text { cancer history }) \\
& +(0.1274 \times \text { diameter }) \\
& +(1.0407 \times \text { spiculation }) \\
& +(0.7838 \times \text { upper lobe })
\end{aligned}
$$

We calculated the diagnostic value of patients with SPN in this study using these 2 models and compared these with plasma ctDNA data.

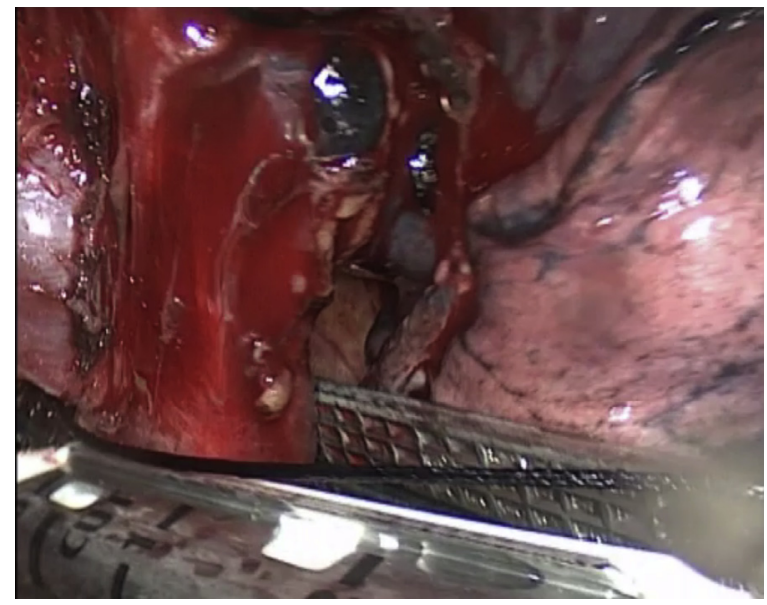

VIDEO 1. VATS right upper lobectomy (partial). Intraoperative plasma was collected before tumor resected. Video available at: http://www. jtcvsonline.org/article/S0022-5223(17)31084-X/addons. 


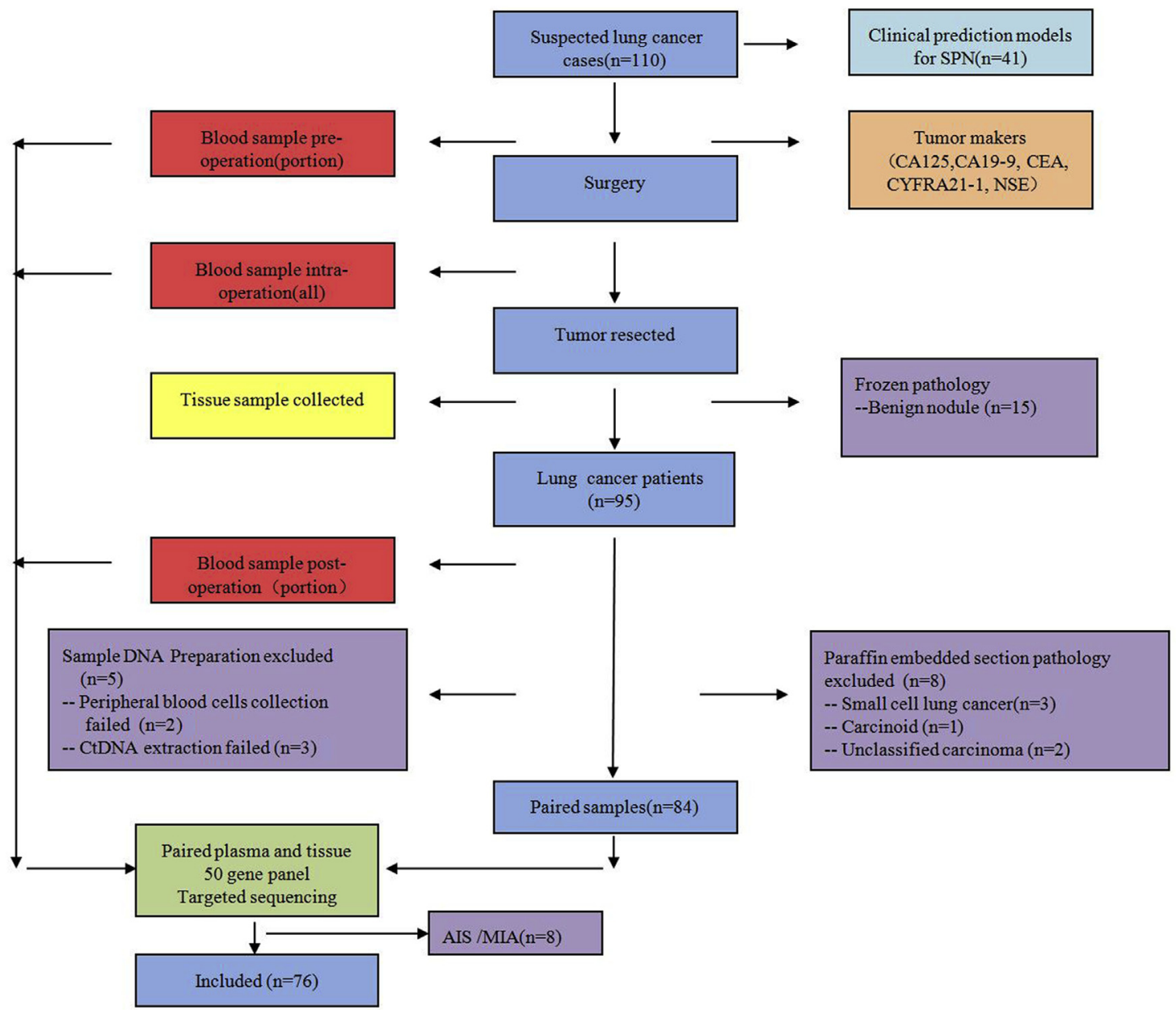

FIGURE 1. Consort diagram. SPN, Solitary pulmonary nodule; CA125, carbohydrate antigen125; CA19-9, carbohydrate antigen19-9; CEA, carcinoembryonic antigen; CYFRA21-1, cytokeratin 19 fragment; NSE, neuron-specific enolase; ctDNA, circulating tumor DNA; AIS, adenocarcinoma in situ; MIA, minimally invasive adenocarcinoma.

\section{Statistical Analysis}

Positively concordant was defined as matched tumor DNA (tDNA) and plasma ctDNA samples with the same identified mutations, and negatively concordant was defined as matched sample pairs without mutations (wild type) in the 50 genes screened. Positive ctDNA therefore was the identification of any mutation in these 50 genes. Categorical data were assessed with the Fisher exact test or Pearson $\chi^{2}$ test. Continuous variables were assessed with the Student $t$ test. Analyses were performed with SPSS Statistics, version 19 (IBM Corp, Chicago, Ill).

\section{RESULTS}

\section{Mutation Concordance in tDNA and Plasma ctDNA Sample Pairs}

Patients' characteristics are shown in Table 1. Of the 76 NSCLC sample pairs analyzed, 55 had mutations in 1 or more of the 50 genes screened in our cancer panel for either tDNA or plasma ctDNA or for both. A total of 31 patients were positively concordant for each mutation, and 21 patients were negatively concordant, yielding an overall concordance of 52 of $76(68.4 \%)$. For the remaining 24 patients, plasma failed to identify tumor tissue mutations in 7 patients, and tumor tissue failed to identify mutations detected by plasma in 7 patients. The other 10 patients had discordant mutations in plasma and tumor tissue. Therefore, 48 patients were positive for ctDNA. No somatic mutations were identified in white blood cell samples, indicating low backgrounds in sequencing reads and variant calling reliability. Detailed results according to TNM stage are shown in Table E1, and data indicate a gradual increase in concordance rate according to stage (stage I, 57.9\%; stage II, $66.7 \%$; stage III, $90 \%, P=.043$ ). No significant difference in concordance rate occurred for other clinical characteristics, including sex, age, smoking history, histology, tumor size, and lymph node status.

Seventy-six samples harbored 89 mutations in ctDNA and 67 mutations in tDNA; mutations were identified in tDNA and/or plasma ctDNA samples in 16 of the 50 genes screened in the cancer panel. Collectively, the mutation profiles for both ctDNA and tDNA reflected the already 
TABLE 1. Baseline characteristics of the patients

\begin{tabular}{|c|c|}
\hline Variable & Value \\
\hline Age, $y$, mean \pm SD & $62.6 \pm 10.8$ \\
\hline \multicolumn{2}{|l|}{ Sex, n (\%) } \\
\hline Male & $46(60.5)$ \\
\hline \multicolumn{2}{|l|}{ Smoking history, n (\%) } \\
\hline Yes & $31(40.8)$ \\
\hline \multicolumn{2}{|l|}{ Histology, n (\%) } \\
\hline Adenocarcinoma & $59(77.6)$ \\
\hline Squamous cell carcinoma & $17(22.4)$ \\
\hline \multicolumn{2}{|l|}{ Postoperative stage, n (\%) } \\
\hline Ia & $25(32.9)$ \\
\hline $\mathrm{Ib}$ & $13(17.1)$ \\
\hline II & $18(23.7)$ \\
\hline III & $20(26.3)$ \\
\hline \multicolumn{2}{|l|}{ Surgical approach, n (\%) } \\
\hline Lobectomy/bilobectomy & $66(86.8)$ \\
\hline Wedge/segmentectomy & $10(13.2)$ \\
\hline Tumor size, $\mathrm{cm}$, mean $\pm \mathrm{SD}$ & $3.39 \pm 1.84$ \\
\hline \multicolumn{2}{|l|}{ Lymph node metastasis, n (\%) } \\
\hline Yes & $30(39.5)$ \\
\hline \multicolumn{2}{|l|}{ Tumor location, $\mathrm{n}(\%)$} \\
\hline Left & $35(46.1)$ \\
\hline Upper lobe & $41(53.9)$ \\
\hline SPN & 31 \\
\hline
\end{tabular}

$S D$, Standard deviation; $S P N$, solitary pulmonary nodule.

published mutation tumor frequencies for lung cancer (Figure 2). ${ }^{12,13}$ Epidermal growth factor receptor (EGFR) was the most common mutation, and detailed distributions of ctDNA and tDNA mutations appear in Figure E2. Table 2 shows a correlation between clinical characteristics

\section{The proportion of tDNA} mutation

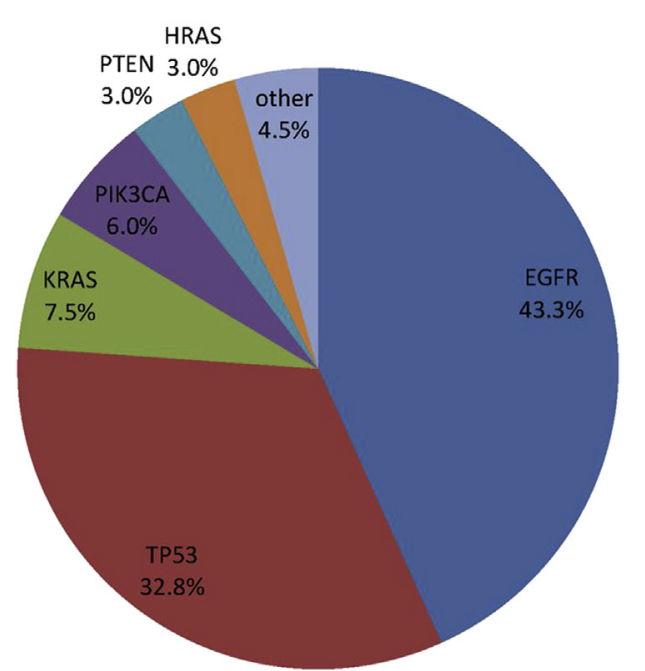

and ctDNA in 76 patients. For patients with tumors exceeding $3 \mathrm{~cm}$ or with lymph node metastasis, ctDNA frequency was significantly greater.

There was no correlation in mutation frequency between primary tumors and matched ctDNA. In tDNA-positive subjects, the average mutation frequency in primary tumors was not significantly different whether matched ctDNA was positive or negative $(31.0 \% \pm 26.0 \%$ vs $20.4 \% \pm 21.5 \%$, $P=.32)$.

\section{Reproducibility and Dynamic Changes in Plasma ctDNA Mutation Identification}

To test reproducibility and dynamic changes we compared plasma ctDNA for 3 time points (Figure 3). For 20 patients who had plasma samples for these time points, 17 had lung cancer and among them, 10 patients were ctDNA positive and 14 mutations were identified. One patient had 3 concomitant mutations (2 different EGFR mutations and 1 PIK3CA mutation), 2 patients had 2 concomitant mutations (EGFR and KRAS; KRAS and TP53). The other 7 patients had 1 mutation each, including 1 EGFR, 2 PTEN, 2 TP53, 1 PIK3CA, and 1 SMAD4. All ctDNA samples obtained pre- and intrasurgery had consistent mutations. The average mutation frequency variance between samples taken pre- and intrasurgery was $1.21 \%(7.94 \% \pm 4.78 \%$ vs $9.15 \% \pm 6.51 \%, P=.58)$. Such low variance in mutation frequency suggests that plasma ctDNA offers reliable and consistent information.

The average postoperation plasma ctDNA mutation frequency was reduced to $0.28 \% \pm 0.32 \%$, which is

\section{The proportion of ctDNA mutation}

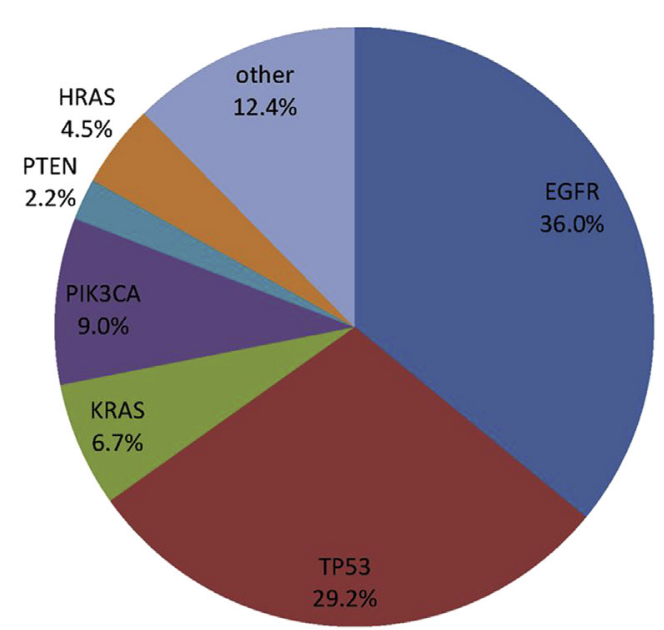

FIGURE 2. Gene mutations identified in tDNA and plasma ctDNA. $t D N A$, Tumor DNA; $c t D N A$, circulating tumor DNA; HRAS, harvey rat sarcoma viral oncogene; PTEN, phosphatase and tensin homolog; PIK3CA, phosphatidylinositol-4,5-bisphosphate 3-kinase catalytic subunit alpha; KRAS, kirsten rat sarcoma viral oncogene; EGFR, epidermal growth factor receptor; TP53, tumor protein p53. 
TABLE 2. Correlation between clinical characteristics and ctDNA detection

\begin{tabular}{|c|c|c|c|c|c|}
\hline \multirow[b]{2}{*}{ Characteristics } & \multicolumn{2}{|c|}{ ctDNA $(\%)$} & \multirow[b]{2}{*}{$P$ value } & $\operatorname{ctDNA}, \% \pm \mathrm{SD}$ & \multirow[b]{2}{*}{$P$ value } \\
\hline & $(+)$ & $(-)$ & & Frequency & \\
\hline \multicolumn{6}{|l|}{ Age, y } \\
\hline$<70$ & $35(63.6)$ & $20(36.4)$ & .89 & $8.5 \pm 15.3$ & .24 \\
\hline$>70$ & $13(61.9)$ & $8(38.1)$ & & $3.4 \pm 4.2$ & \\
\hline \multicolumn{6}{|l|}{ Sex } \\
\hline Male & $30(65.2)$ & $16(34.8)$ & .65 & $5.6 \pm 10.5$ & .30 \\
\hline Female & $18(60)$ & $12(40)$ & & $9.8 \pm 17.2$ & \\
\hline \multicolumn{6}{|l|}{ Smoking history } \\
\hline Smoking & $19(61.3)$ & $12(38.7)$ & .78 & $8.0 \pm 12.6$ & .72 \\
\hline Nonsmoking & $29(64.4)$ & $16(35.6)$ & & $6.6 \pm 14.1$ & \\
\hline \multicolumn{6}{|l|}{ Histology, n (\%) } \\
\hline Adenocarcinoma & $37(62.7)$ & $22(37.3)$ & .88 & $6.9 \pm 13.6$ & .82 \\
\hline Squamous & $11(64.7)$ & $6(35.3)$ & & $8.0 \pm 13.3$ & \\
\hline \multicolumn{6}{|l|}{ Tumor size } \\
\hline$>3 \mathrm{~cm}$ & $21(65.6)$ & $11(34.4)$ & .70 & $11.7 \pm 19.0$ & .038 \\
\hline$<3 \mathrm{~cm}$ & $27(61.4)$ & $17(38.6)$ & & $3.6 \pm 4.4$ & \\
\hline \multicolumn{6}{|l|}{$\mathrm{N}$ stage } \\
\hline No & $28(60.9)$ & $18(39.1)$ & .61 & $3.8 \pm 4.6$ & .037 \\
\hline $\mathrm{N} 1 / \mathrm{N} 2$ & $20(66.7)$ & $10(33.3)$ & & $11.9 \pm 19.4$ & \\
\hline \multicolumn{6}{|l|}{ Stage } \\
\hline I & $25(65.8)$ & $13(34.2)$ & .63 & $4.1 \pm 4.7$ & .097 \\
\hline $\mathrm{II} / \mathrm{III}$ & $23(60.5)$ & $15(39.5)$ & & $10.5 \pm 18.4$ & \\
\hline
\end{tabular}

ctDNA, Circulating tumor DNA; $S D$, standard deviation.

significantly lower than before surgery $(P<.001)$. All the identified plasma ctDNA mutations had a decrease in mutation frequency after surgery, and $5(36 \%)$ mutations decreased to zero. The detailed frequency variances are shown in Table E2.

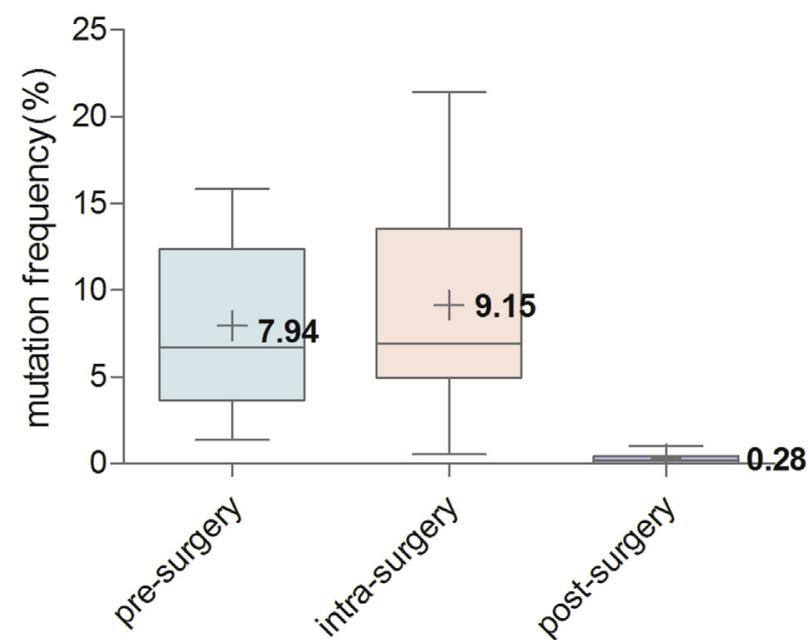

FIGURE 3. Box-and-whisker plot of mutation frequencies of somatic mutations detected in plasma ctDNA pre-, intra-, and postsurgery. The horizontal lines of each box from bottom to top were the min, first quartile, median, third quartile, and max value, respectively. The plus represents the mean value. Ten patients were compared in the boxes.

\section{Preliminary Clinical Exploration of ctDNA in Early Diagnosis of NSCLC}

To assess the potential application for early diagnosis, we compared ctDNA with tumor markers and radiographicbased clinical predictive models. Tumor markers including carcinoembryonic antigen, carbohydrate antigen 19-9, carbohydrate antigen 125, cytokeratin 19 fragment, and neuron-specific enolase in serum are commonly used markers for diagnosis or surveillance of patients with lung cancer. In this study, most patients were analyzed for plasma tumor markers before surgery, and they were considered negative based on established thresholds (carbohydrate antigen $125<35 \mathrm{U} / \mathrm{mL}$, carbohydrate antigen $19-9<39 \mathrm{U} / \mathrm{mL}$, carcinoembryonic antigen $<4.7 \mathrm{ng} / \mathrm{mL}$, cytokeratin 19 fragment $<3.3 \mathrm{ng} / \mathrm{mL}$, neuron-specific enolase $<16.3 \mathrm{ng} / \mathrm{mL})$. Thirty-six patients $(49.3 \%)$ were positive for one or more tumor markers analyzed. In contrast, 48 patients $(63.2 \%)$ were ctDNA positive. ctDNA was more sensitive for lung cancer compared with the other 5 tumor markers and all tumor markers overall (Table 3 ). A total of 31 patients with lung cancer were considered as SPN in computed tomography (CT) scan, whereas in the 15 benign patients, 10 were SPN. Therefore, 41 patients with SPN were included to compare the prediction value between plasma ctDNA and clinical models. The receiver operating characteristic curves are shown in Figure 4. The area under curves was 0.887 by ctDNA (95\% confidence interval [CI], 0.788-0.986), 0.803 by 
TABLE 3. Detection of plasma ctDNA versus tumor markers CA125, CA19-9, CEA, CYFRA21-1, and NSE

\begin{tabular}{lccc}
\hline \multicolumn{1}{c}{ Marker } & Positive samples & Total samples & Sensitivity \\
\hline ctDNA & 48 & 76 & $63.2 \%$ \\
CA125 & 5 & 65 & $7.7 \%$ \\
CA19-9 & 3 & 70 & $4.3 \%$ \\
CEA & 19 & 72 & $26.4 \%$ \\
CYFRA21-1 & 24 & 71 & $33.8 \%$ \\
NSE & 10 & 71 & $14.1 \%$ \\
All Tumor marker & 36 & 73 & $49.3 \%$ \\
\hline
\end{tabular}

ctDNA, Circulating tumor DNA; CA125, carbohydrate antigen125; CA19-9, carbohydrate antigen 19-9; CEA, carcinoembryonic antigen; CYFRA21-1, cytokeratin 19 fragment; $N S E$, neuron-specific enolase.

PKU model (95\% [CI], 0.647-0.959), and 0.69 by Mayo model (95\% CI, 0.512-0.869), respectively. No benign SPN was positive in ctDNA detection, which indicated a high specificity to predict malignancy of SPN by ctDNA detection.

\section{DISCUSSION}

Few studies on ctDNA focus on stage I-IIIA surgical patients and most ctDNA studies only refers to a few driver mutations such as EGFR or KRAS. ${ }^{14,15}$ However, many patients lack these mutations, limiting its applicability in that only a minority of patients could be detected plasma ctDNA. Guo and colleagues ${ }^{16}$ measured EGFR mutations in plasma in 198 patients with NSCLC, but only 34 patients were positive. We prospectively collected plasma and tumor tissue from surgical patients with NSCLC and compared multiple gene mutations by using an NGS-based 50-gene panel, which did not require patient-specific optimization and covered most patients with NSCLC, therefore this may increase the clinical utility of ctDNA.

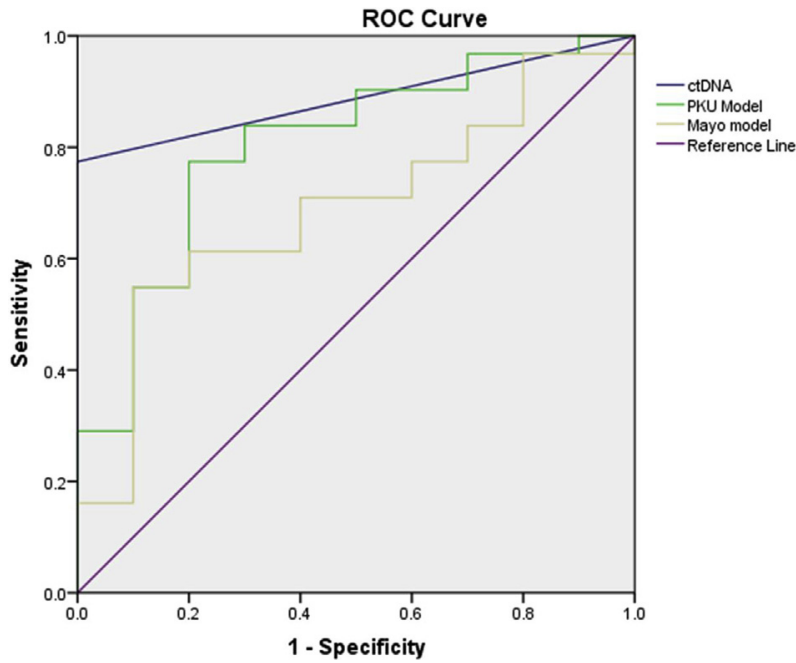

FIGURE 4. Comparison of ROC curves using the ctDNA, PKU, and Mayo models. ROC, Receiver operating characteristic; $c t D N A$, circulating tumor DNA.
Previous studies indicate that concordance for multiple genes in matched tDNA and ctDNA was $76.2 \%$ for patients with advanced-stage lung cancer. ${ }^{8}$ Our results show that for patients with early and locally advanced lung cancer, concordance mainly depends on tumor stage. Stage III patients had greater concordance than stage I patients and in the patients $(35.4 \%, 17 / 48)$ who failed to identify tumor tissue mutations in plasma ctDNA, most $(76.5 \%, 13 / 17)$ were stage I-IIA. This result is similar to the report of Newman and colleagues, ${ }^{4}$ in which ctDNA mutations were confirmed in only $50 \%$ of patients with stage I-IIA cancer, indicating that ctDNA measurement is feasible for surgical patients with lung cancer, but more sensitive methods are required for patients with early-stage cancer.

Discrepancies in mutations in plasma ctDNA but not in tDNA may be due to intra- and inter-tumor heterogeneity. Jamal-Hanjani and colleagues ${ }^{17}$ compared heterogeneous mutations in 4 patients with early-stage NSCLC and of the 16 variants found in ctDNA, 4 were heterogeneous variants based on the previous intratumor sequencing area. Because we collected samples prospectively, most tissue should send to paraffin-embedded pathologic examination. We could only obtain fresh tissue samples by excising a small patch from the core tumor component, not excising the entire tumor. Therefore, only a portion of the tumor was used for sequencing analysis and may not represent entire tumor features. ${ }^{18,19}$ In contrast, 14 patients with lung cancer in this study had scattered small GGO according to CT scans, which may be considered as multifocal primary lung cancers. Two patients were positively and 2 patients were negatively concordant, and in 1 patient the tumor mutation wasn't identified in plasma. Of the other 9 patients, plasma ctDNA identified additional mutations not found in paired tDNA, indicating that only $28.6 \%$ patients with lung cancer with scattered GGO were concordant in ctDNA and tDNA detection. These small lung cancer lesions do not require removal according to guidelines, ${ }^{20}$ but intertumor heterogeneity among GGOs and primary lesions may offer different mutation profiles to explain discordant plasma ctDNA and tDNA data in this study. Izumchenko and colleagues found a BRAF mutation in plasma ctDNA identified in premalignant atypical adenomatous hyperplasia lesion, which suggested the promise for ctDNA in real-time sampling of multifocal clonal evolution and provided evidence that genetic alterations associated with very small early neoplasms can be detected in paired ctDNA. ${ }^{21}$ Therefore, ctDNA may be more representative of the holistic tumor burden than tDNA but more studies are needed to validate this.

Of the subjects enrolled, 21 had no mutations in both ctDNA and tDNA, perhaps because we only studied 50 genes in a panel that covered single-nucleotide 
polymorphisms, insertions, and deletions but not rearrangements such as EML4-ALK or ROS1. Therefore, although we included many alterations, it was not sufficiently broad to cover all NSCLC patients. Newman and colleagues, ${ }^{4}$ the authors identified mutations in $>95 \%$ tumors using a 139-gene panel. We admitted a more comprehensive panel could have more clinical value, but the cost and technical challenges for fusion gene detection should be taken into consideration.

Patients with more invasive tumor status had significantly greater ctDNA frequency. However, ctDNA mutation frequency was not correlated to tDNA frequency. Possible explanation may come from intratumor tumor cell apoptosis rate and variability in the distribution of mutated cells throughout the tissue. ${ }^{21}$

To investigate the potential diagnostic and monitoring value, we compared plasma ctDNA by different time points. Theoretically, it would be more matching if plasma and tissue samples were obtained at the same time. Indeed, we found that plasma samples obtained 1 to 3 days before surgery and during surgery had the same mutations with a low variance in mutation frequency, indicating reproducibility of plasma ctDNA mutation identification using our method. Assessing presurgical samples is of greater clinical value, as it may guide treatment choices before surgery begins.

Randomized controlled trials are underway to define a role for EGFR TKIs as neoadjuvant treatment for surgical NSCLC with EGFR activated mutations. ${ }^{22}$ However, these trials are hampered by tissue sample limitations. Some samples are difficult to obtain and small tumors sampled with endobronchial ultrasound or CT-guided puncture may cause sampling bias (intratumor heterogeneity) which limits identification of gene mutation status. ${ }^{23}$ Diverse outcomes for trials of neoadjuvant targeted treatment suggest that limitations of gene detection are imposed by tumor tissue. $^{24,25}$ Plasma ctDNA may overcome time and space heterogeneity and represent gene mutations for the entire human body. ${ }^{26}$ Also, plasma ctDNA collection is noninvasive and more convenient than tumor tissue, with consistent results pre- and intraoperatively, and it may be more reliable and practical for guiding TKI neoadjuvant treatment based on data obtained from plasma ctDNA.

Studies have shown that ctDNA degrades in 15 minutes to 2 hours after surgery, ${ }^{1}$ and we noted that plasma ctDNA mutation frequency was significantly decreased 1 day after surgery. However, only some of the mutations decrease to zero, and these data agree with the work of Diehl and colleagues, $^{27}$ which showed $20 \%$ patients' ctDNA decreased to 0 at 24 hours after surgery of rectal carcinoma. This may be the reason why some patients with early-stage lung cancer appear early recurrence after surgery. Monitoring plasma ctDNA may be an approach to monitor residual tumors after surgery.
Early diagnosis is paramount for patients with lung cancer, ${ }^{28}$ but convenient and occasionally used tumor markers have been questioned for diagnosing lung cancer due to lack of sensitivity and specificity, because they can be elevated during noncancerous events. ${ }^{29}$ We found that plasma ctDNA was more sensitive and may be used as a substitution of tumor markers for initial screening of lung cancer. Comparing plasma ctDNA and 2 clinical mathematical prediction models indicate that plasma ctDNA was more predicative for diagnosing of SPN. No patients with benign lesions were positive for ctDNA detection, indicating that if ctDNA is positive, the nodule will likely be malignant, and aggressive approaches for diagnosis and management is recommended. Therefore, ctDNA may offer additional information than clinical features at diagnosis of lung cancer. However, because of the small sample size of this study, larger sample size studies with internal and external validation is needed to confirm this. In addition, this study is limited by the simplex analysis of mutations. Considering a multimarker approach may offer a more comprehensive insight into patients with cancer. For example, epigenetic analysis for detection of aberrant methylation in ctDNA may provide information about the tumor microenvironment, which usually lacks somatic mutations, and could potentially be further improved if combined with mutational analysis. ${ }^{30}$

In summary, this prospective study showed multiple genes detection using plasma ctDNA in surgical patients with NSCLC is feasible. Although technical and biological challenges remain, ctDNA presents a potential for clinical management. Further studies are needed before this is brought into mainstream clinical practice.

\section{Webcast}

You can watch a Webcast of this AATS meeting presentation by going to: http://webcast.aats.org/2016/Video/ Tuesday/05-17-16_Room_343_0710_Chen-800.mp4.

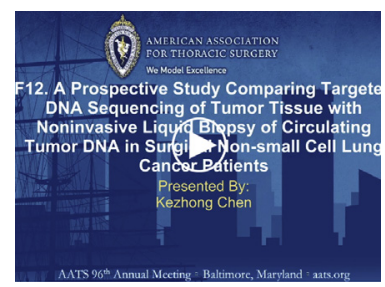

\section{Conflict of Interest Statement}

Authors have nothing to disclose with regard to commercial support.

\section{References}

1. Bettegowda C, Sausen M, Leary RJ, Kinde I, Wang Y, et al. Detection of circulating tumor DNA in early- and late-stage human malignancies. Sci Transl Med. 2014;6:224ra224. 
2. Lim E, Tay A, Von Der Thusen J, Freidin MB, Anikin V, Nicholson AG, et al Clinical results of microfluidic antibody-independent peripheral blood circulating tumor cell capture for the diagnosis of lung cancer. $J$ Thorac Cardiovasc Surg. 2014;147:1936-8.

3. Heitzer E, Ulz P, Geigl JB. Circulating tumor DNA as a liquid biopsy for cancer. Clin Chem. 2015;61:112-23.

4. Newman AM, Bratman SV, To J, Wynne JF, Eclov NC, Modlin LA, et al. An ultrasensitive method for quantitating circulating tumor DNA with broad patient coverage. Nat Med. 2014;20:548-54.

5. Narayan A, Carriero NJ, Gettinger SN, Kluytenaar J, Kozak KR, Yock TI, et al Ultrasensitive measurement of hotspot mutations in tumor DNA in blood using error-suppressed multiplexed deep sequencing. Cancer Res. 2012;72: 3492-8.

6. Garcia-Murillas I, Schiavon G, Weigelt B, Ng C, Hrebien S, Cutts RJ, et al. Mutation tracking in circulating tumor DNA predicts relapse in early breast cancer. Sci Transl Med. 2015;7:302ra133.

7. Bai X, Zhang E, Ye H, Nandakumar V, Wang Z, Chen L, et al. PIK3CA and TP53 gene mutations in human breast cancer tumors frequently detected by ion torrent DNA sequencing. PLoS One. 2014;9:e99306.

8. Xu S, Lou F, Wu Y, Sun DQ, Zhang JB, Chen W, et al. Circulating tumor DNA identified by targeted sequencing in advanced-stage non-small cell lung cancer patients. Cancer Lett. 2016;370:324-31.

9. Gould MK, Donington J, Lynch WR, Mazzone PJ, Midthun DE, Naidich DP, et al. Evaluation of individuals with pulmonary nodules: when is it lung cancer? Diagnosis and management of lung cancer, 3rd ed: American College of Chest Physicians evidence-based clinical practice guidelines. Chest. 2013;143: e93S-120S.

10. Swensen SJ, Silverstein MD, Edell ES, Trastek VF, Aughenbaugh GL, Ilstrup DM, et al. Solitary pulmonary nodules: clinical prediction model versus physicians. Mayo Clin Proc. 1999;74:319-29.

11. Li Y, Chen KZ, Wang J. Development and validation of a clinical prediction model to estimate the probability of malignancy in solitary pulmonary nodules in Chinese people. Clin Lung Cancer. 2011;12:313-9.

12. An SJ, Chen ZH, Su J, Zhang XC, Yang JJ, Zhou Q, et al. Identification of enriched driver gene alterations in subgroups of non-small cell lung cancer patients based on histology and smoking status. PLoS One. 2012;7:e40109.

13. Shi Y, Au JS, Thongprasert S, Srinivasan S, Tsai CM, Khoa MT, et al. A prospective, molecular epidemiology study of EGFR mutations in Asian patients with advanced non-small-cell lung cancer of adenocarcinoma histology (PIONEER). J Thorac Oncol. 2014;9:154-62.

14. Mok T, Wu YL, Lee JS, Yu CJ, Sriuranpong V, Sandoval-Tan J, et al. Detection and dynamic changes of EGFR mutations from circulating tumor DNA as a predictor of survival outcomes in NSCLC patients treated with first-line intercalated erlotinib and chemotherapy. Clin Cancer Res. 2015;21:3196-203.

15. Rosell R, Karachaliou N. Lung cancer: Using ctDNA to track EGFR and KRAS mutations in advanced-stage disease. Nat Rev Clin Oncol. 2016;13:401-2.

16. Guo K, Zhang Z, Han L, Han J, Wang J, Zhou Y, et al. Detection of epidermal growth factor receptor mutation in plasma as a biomarker in Chinese patients with early-stage non-small cell lung cancer. Onco Targets Ther. 2015;8: 3289-96.

17. Jamal-Hanjani M, Wilson GA, Horswell S, Mitter R, Sakarya O, et al. Detection of ubiquitous and heterogeneous mutations in cell-free DNA from patients with early-stage non-small-cell lung cancer. Ann Oncol. 2016;27:862-7.

18. Gerlinger M, Rowan AJ, Horswell S, Larkin J, Endesfelder D, et al. Intratumor heterogeneity and branched evolution revealed by multiregion sequencing, N Engl J Med. 2012;366:883-92.

19. Swanton C. Intratumor heterogeneity: evolution through space and time. Cancer Res. 2012;72:4875-82.

20. Naidich DP, Bankier AA, MacMahon H, Schaefer-Prokop CM, Pistolesi M, Goo JM, et al. Recommendations for the management of subsolid pulmonary nodules detected at CT: a statement from the Fleischner Society. Radiology. 2013;266:304-17.

21. Izumchenko E, Chang X, Brait M, Fertig E, Kagohara LT, Bedi A, et al. Targeted sequencing reveals clonal genetic changes in the progression of early lung neoplasms and paired circulating DNA. Nat Commun. 2015;6:8258.

22. Zhai H, Zhong W, Yang X, Wu YL. Neoadjuvant and adjuvant epidermal growth factor receptor tyrosine kinase inhibitor (EGFR-TKI) therapy for lung cancer. Transl Lung Cancer Res. 2015;4:82-93.

23. de Bruin EC, McGranahan N, Mitter R, Salm M, Wedge DC, Yates L, et al. Spatial and temporal diversity in genomic instability processes defines lung cancer evolution. Science. 2014;346:251-6.
24. Schaake EE, Kappers I, Codrington HE, Valdes RA, Teertstra HJ, et al. Tumor response and toxicity of neoadjuvant erlotinib in patients with early-stage non-small-cell lung cancer. J Clin Oncol. 2012;30:2731-8.

25. Lara-Guerra H, Waddell TK, Salvarrey MA, Joshua AM, Chung CT, Paul N, et al. Phase II study of preoperative gefitinib in clinical stage I non-small-cell lung cancer. J Clin Oncol. 2009;27:6229-36.

26. Murtaza M, Dawson SJ, Pogrebniak K, Rueda OM, Provenzano E, Grant J, et al. Multifocal clonal evolution characterized using circulating tumour DNA in a case of metastatic breast cancer. Nat Commun. 2015;6:8760.

27. Diehl F, Schmidt K, Choti MA, Romans K, Goodman S, Li M, et al Circulating mutant DNA to assess tumor dynamics. Nat Med. 2008;14 985-90.

28. Reddy RM, Murlidhar V, Zhao L, Grabauskiene S, Zhang Z, Ramnath N, et al. Pulmonary venous blood sampling significantly increases the yield of circulating tumor cells in early-stage lung cancer. J Thorac Cardiovasc Surg. 2016;151:852-7.

29. Ma S, Shen L, Qian N, Chen K. The prognostic values of CA125, CA19.9, NSE, AND SCC for stage I NSCLC are limited. Cancer Biomark. 2011;10: 155-62.

30. Wan JC, Massie C, Garcia-Corbacho J, Mouliere F, Brenton JD, Caldas C, et al. Liquid biopsies come of age: towards implementation of circulating tumour DNA. Nat Rev Cancer. 2017;17:223-38.

Key Words: lung cancer, circulating tumor DNA, surgery

\section{Discussion}

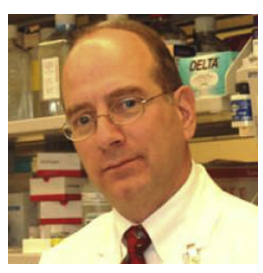

Dr D. Schrump (Bethesda, Md). Congratulations on this very important study.

The fact that plasma DNA has a half-life of 2 hours really suggests that analysis of circulating tumor DNA may prove very useful for early detection and monitoring treatment responses in patients with lung cancer.

I have several questions. It seemed that there were a lot of nonsmokers, which you might expect in your population. Given the fact that the mutational load in smokers is greater than nonsmokers, have you had an opportunity to look at whether the levels of circulating tumor DNA were greater in the smokers relative to the nonsmokers?

Dr Kezhong Chen (Beijing, China). Thank you. It's a very good question.

Yes, we have the data comparing the nonsmokers and the smokers. In China, the mutation frequency is greater in nonsmokers than smokers, because in Chinese people now, there are more and more patients with cancer who are female, nonsmokers, and younger, multiple GGO patients. These patients have a greater frequency of mutation.

Dr Schrump. EGFR mutations?

Dr Chen. Yes, EGFR mutations.

Dr Schrump. But I'm talking about other mutations.

Dr Chen. The other mutations are not a high frequency. EGFR may be $50 \%$, but other mutations may be less than $10 \%$ in this sample. It's not a big sample. There were only 76 patients. We cannot conclude the results of other mutations.

Dr Schrump. It looked like you had very high concordance between tumor and circulating DNA as far as EGFR and p53.

Dr Chen. Yes. 
Dr Schrump. And really the low concordance seemed to be associated with genes that had very low mutational frequency, and I just wonder if you remove some of those from your analysis whether or not your concordance rate would be significantly greater.

Dr Chen. In fact, we used the white blood cells as a control. In the white blood cells, the germline gene mutations were excluded. And the low frequency, we have a cutoff value. If the frequency was less than $0.5 \%$, we excluded it.
Dr Schrump. There are a number of genes that are methylated in virtually $100 \%$ of lung cancers, and I wonder if you have started to look at any of these, such as HOXA9, SOX17, and so forth. Inclusion of some of these methylated genes might improve your ability to detect early stage lung cancers.

Dr Chen. Yes, a very good question. Yes, we plan to do some other studies. We are doing a study comparing other biomarkers. It is a prospective study that has been registered in ClinicalTrials.gov. 


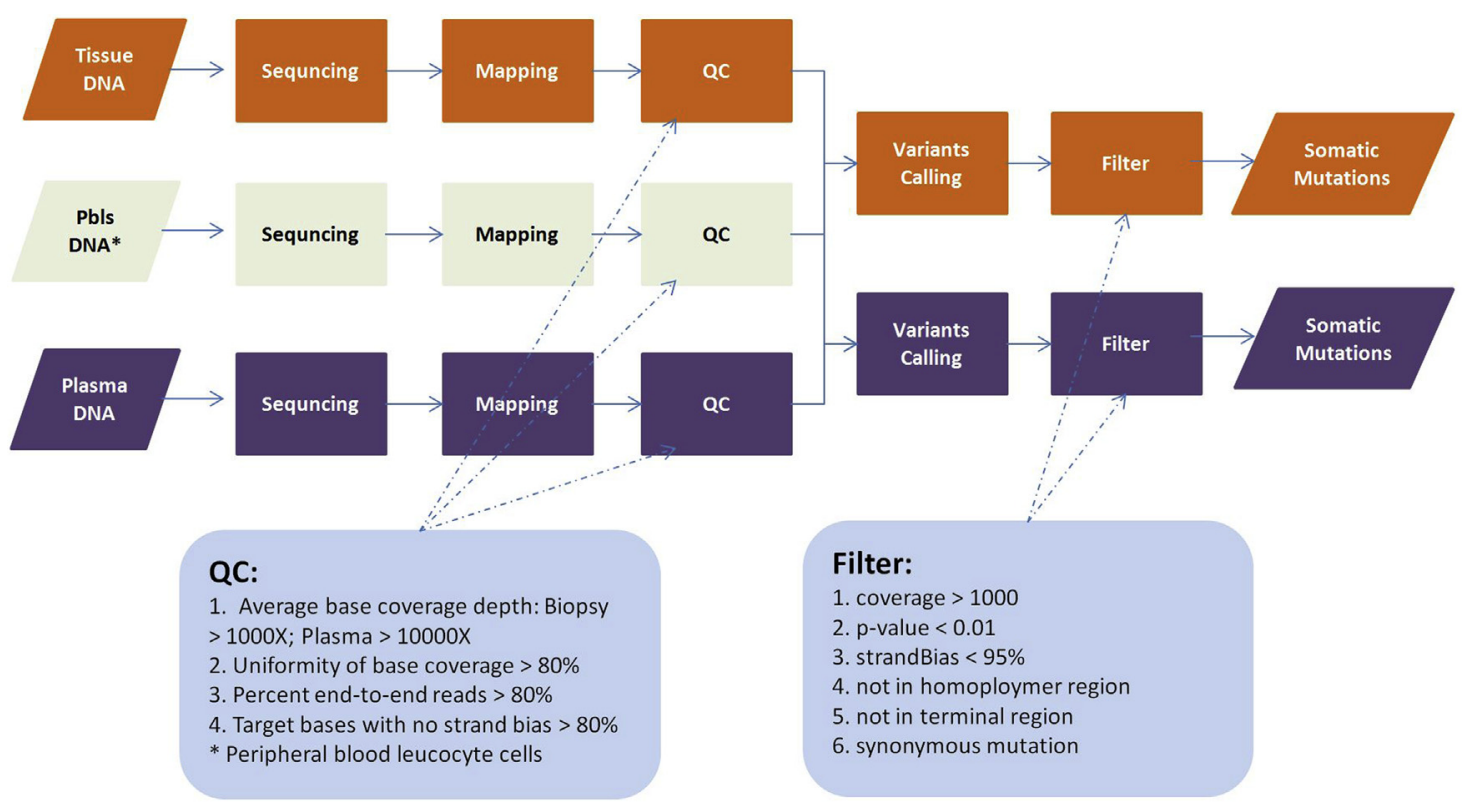

FIGURE E1. Measurement procedure and quality control. $Q C$, Quality control.
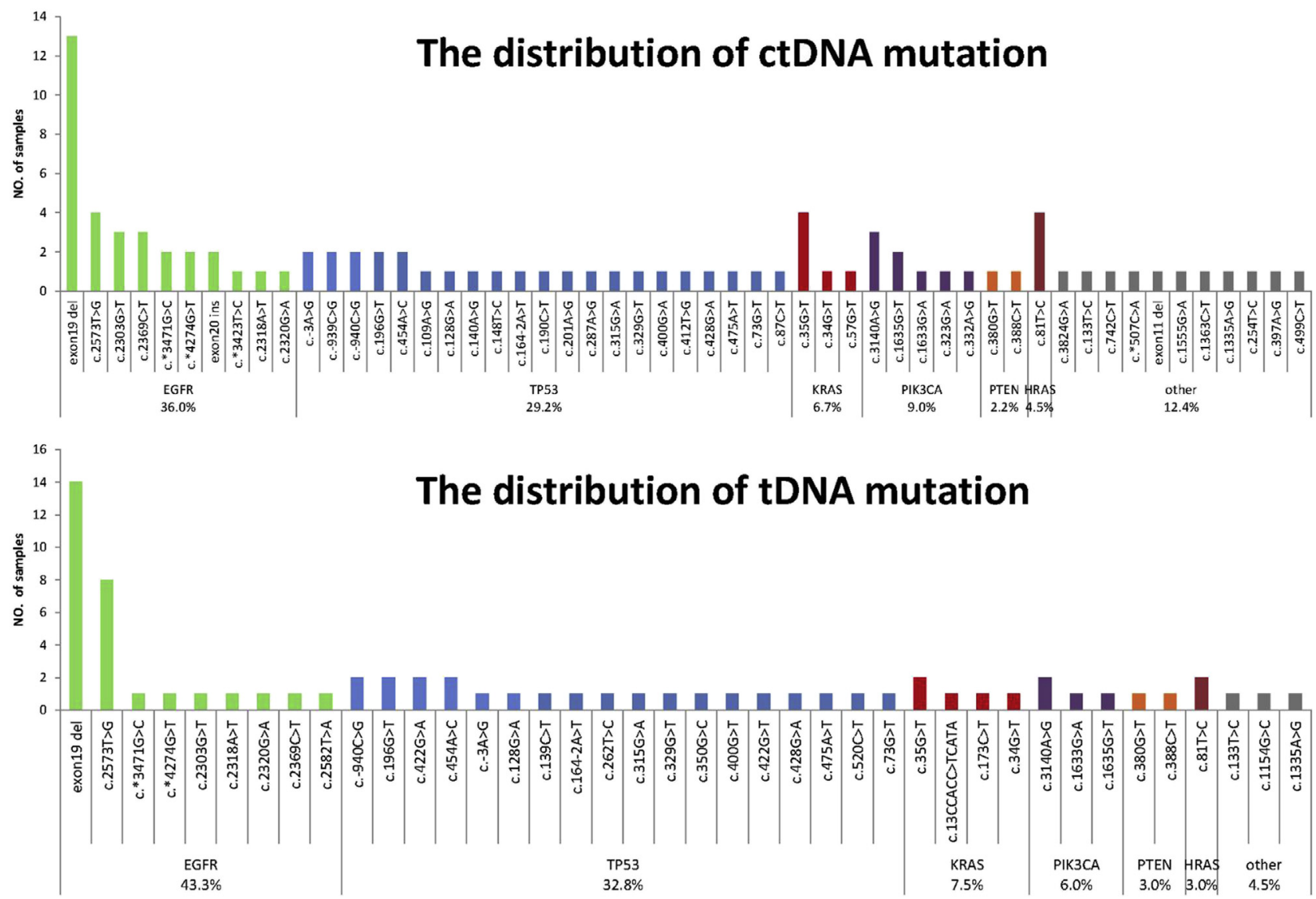

FIGURE E2. The detailed distribution of ctDNA and tDNA mutations. $c t D N A$, Circulating tumor DNA; $t D N A$, tumor DNA; EGFR, epidermal growth factor receptor; TP53, tumor protein p53; KRAS, kirsten rat sarcoma viral oncogene; PIK3CA, phosphatidylinositol-4,5-bisphosphate 3-kinase catalytic subunit alpha; PTEN, phosphatase and tensin homolog; HRAS, harvey rat sarcoma viral oncogene. 
TABLE E1. Detailed results comparing ctDNA and tDNA according to TNM stage

\begin{tabular}{lcccc}
\hline \multicolumn{1}{c}{ Result } & Stage I & Stage II & Stage III & Total \\
\hline Positively concordant & 14 & 6 & 11 & 31 \\
Negatively concordant & 8 & 6 & 7 & 21 \\
Tissue positive & 5 & 1 & 1 & 7 \\
Plasma positive & 6 & 1 & 0 & 7 \\
Both discordant & 5 & 4 & 1 & 10 \\
Tissue positive: patients had mutations identified in tDNA but not in ctDNA; plasma \\
positive: patients had mutations identified in ctDNA but not in tDNA; both discordant: \\
patients had discordant mutations in both ctDNA and tDNA.
\end{tabular}

TABLE E2. Variance frequency for ctDNA at 3 time points

\begin{tabular}{llccc}
\hline & & \multicolumn{3}{c}{ Mutation frequency } \\
\cline { 3 - 5 } Patients & Mutations & $\begin{array}{c}\text { Preoperation } \\
\%\end{array}$ & $\begin{array}{c}\text { Intraoperation } \\
\%\end{array}$ & $\begin{array}{c}\text { Postoperation } \\
\%\end{array}$ \\
\hline 1 & EGFR & 4.69 & 8.22 & 0.41 \\
& KRAS & 2.81 & 3.13 & 0.11 \\
2 & EGFR ${ }^{*}$ & 3.62 & 5.99 & 0.1 \\
& EGFR $\dagger$ & 3.68 & 3.74 & 0.38 \\
& PIK3CA & 6.47 & 5.6 & 0.32 \\
3 & EGFR & 15.78 & 21.43 & 0 \\
4 & PTEN & 1.37 & 0.53 & 0 \\
5 & PTEN & 6.34 & 5.38 & 0.24 \\
6 & TP53 & 12.27 & 18.43 & 1.02 \\
7 & TP53 & 7.63 & 7.63 & 0 \\
& KRAS & 15.85 & 20 & 0.51 \\
8 & TP53 & 12.64 & 11.89 & 0.81 \\
9 & SMAD4 & 11 & 9.82 & 0 \\
10 & PIK3CA & 6.98 & 6.24 & 0 \\
\hline
\end{tabular}

$\overline{E G F R \text {, Epidermal growth factor receptor; } K R A S \text {, kirsten rat sarcoma viral oncogene; }}$ PIK3CA, phosphatidylinositol-4,5-bisphosphate 3-kinase catalytic subunit alpha; $P T E N$, phosphatase and tensin homolog; TP53, tumor protein p53; SMAD4, mothers against decapentaplegic homolog $4 .{ }^{*} \mathrm{chr} 7:$ 55242464AGGAATTAAGAGAAGC $>$ A. †chr7: $55242511 \mathrm{G}>\mathrm{T}$. 